\section{Stroke in three patients due to spontaneous intra tumoral hemorrhage in meningiomas: Lessons learnt}

Sir,

Barring aneurysmal bleeds, most of spontaneous intracerebral hemorrhage ( $\mathrm{ICH})$ patients are managed conservatively. ${ }^{[1]}$ A rare cause of these bleeds can be a hemorrhage associated with an underlying brain tumor. It is imperative to diagnose this rare category of ICH promptly because the main-stay of treatment is surgical evacuation that gives good results. The situation gets further trickier in those cases where intra tumoral hemorrhage (ITH) is the presenting event in a hitherto unknown tumor.

We report three cases of ITH in meningiomas, primarily presenting clinically as sudden neurological deterioration and hence mimicking stroke.
First case was a 55-year-old female patient presented with sudden onset severe headache, followed by loss of consciousness. She was on aspirin prophylaxis for coronary artery disease. Her Glasgow Coma Scale (GCS) was $9\left(\mathrm{E}_{2} \mathrm{~V}_{2} \mathrm{M}_{5}\right)$ with bilateral papilledema and right hemiparesis. Non-contrast computed tomography (NCCT) head revealed a deep-seated bleed on the left side (? Capsulo ganglionic bleed) [Figure 1a]. Further probing revealed a history of gradually progressive right hemiparesis of 2 months duration and magnetic resonance imaging (MRI) was done, which showed a large, extra-axial lesion arising from the middle one-third of the falx on the left side causing a mass effect. The lesion was of variable intensity [Figure 1b-e]. A diagnosis of the middle one-third of falcine meningioma with intratumoral bleed was made. The therapeutic plan shifted dramatically and urgent surgical excision of the tumor with evacuation of bleed was undertaken. The intra-operatively tumor was grayish, soft, suckable, and highly vascular, with areas of the bleed present. Post operative CT revealed complete tumor excision [Figure 1f] Histopathology was suggestive of meningothelial meningioma (WHO grade I).

The second case was a 72-year-old female patient presented with sudden onset severe headache, followed

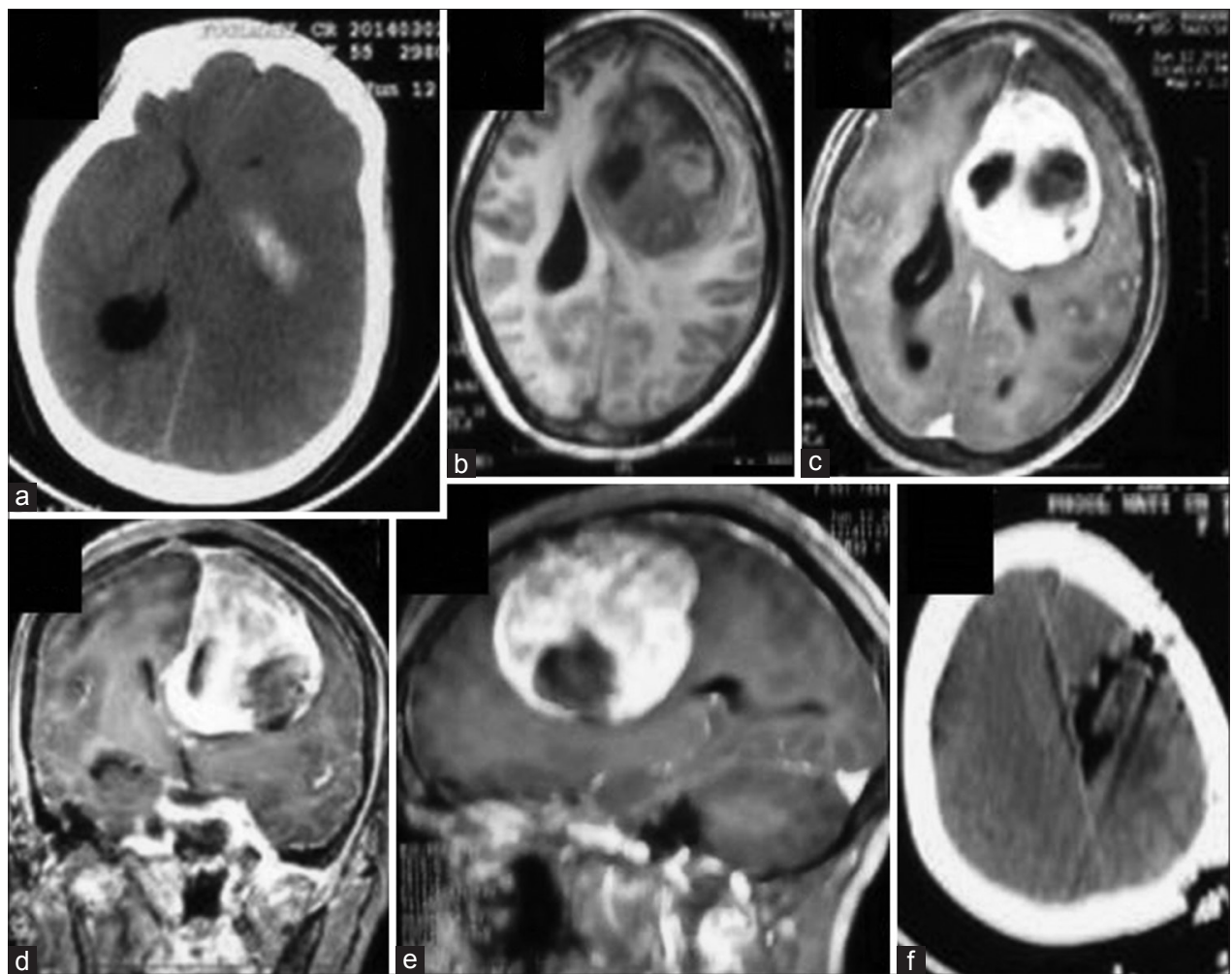

Figure 1: (a) Non contrast computed tomography head demonstrating a largely isointense lesion in left frontal region with an area of hyperintensity medially and anteriorly. There was midline shift. (b) T1-weighted magnetic resonance imaging axial showing a large variable intensity lesion in left frontal area (mid $1 / 3^{\text {rd }}$ ) arising from falx. (c-e) (Axial, coronal, sagittal) magnetic resonance imaging T1-weighted imaging after contrast administration shows intense contrast enhancement. (f) Postoperative computed tomography showing complete tumor excision 
by loss of consciousness. She was on aspirin prophylaxis for coronary artery disease. Her GCS was $\mathrm{E}_{4} \mathrm{~V}_{4} \mathrm{M}_{6}$ with bilateral papilledema.

NCCT head revealed an isointense lesion in the left frontotemporal region with areas of the bleed inside [Figure 2a]. She had a history of 2 episodes of Generalized tonic clonic seizures (GTCS) 2 months before admission. MRI showed a large, extra-axial lesion in the lateral sphenoid wing region. The lesion was variable intensity [Figure 2b-e]. A diagnosis of lateral one-third sphenoid wing meningioma with intratumoral bleed was kept. The intra-operatively tumor was grayish, soft, suckable, and highly vascular, with areas of the bleed present. Histopathology was suggestive of meningiothelial meningioma (WHO grade I).

The third case was 25-year-old male presented with sudden onset severe holocranial headache, associated with vomiting and visual blurring. NCCT head [Figure 3a] showed an extra-axial hyperdense lenticular lesion in the right frontotemporal region. On first look, the lesion appeared to be an extradural hematoma (EDH). On persistent probing, a history of occasional headaches was present. This coupled with absence of history of trauma compelled us to think of some other underlying pathology and an MRI was sought which demonstrated a dural-based, extra-axial, lesion, isointense on T1- and T2-weighted images, with a central area of variable intensity (? convexity meningioma with bleed) [Figure 3b-f]. The lesion, barring the central area of variable intensity, demonstrated homogeneous contrast enhancement. The patient underwent emergency craniotomy and gross total excision of the tumor with the evacuation of hematoma. The patient is doing fine at 6 months follow-up.

Spontaneous ICH (especially deep-seated ones) most of the times does not merit surgical evacuation because it does not improve the prognosis. A rare cause of spontaneous ICH is hemorrhage associated with an underlying brain tumor. The management of these bleeds is diametrically opposite as surgical evacuation gives good results.

Spontaneous ITH in meningioma is an uncommon occurrence $(1.3-2.4 \%) .{ }^{[2]}$ Still rare is the presentation where ITH is the first presenting event.

We want to highlight that in cases of ITH in meningiomas, there is a need for prompt and accurate diagnosis and

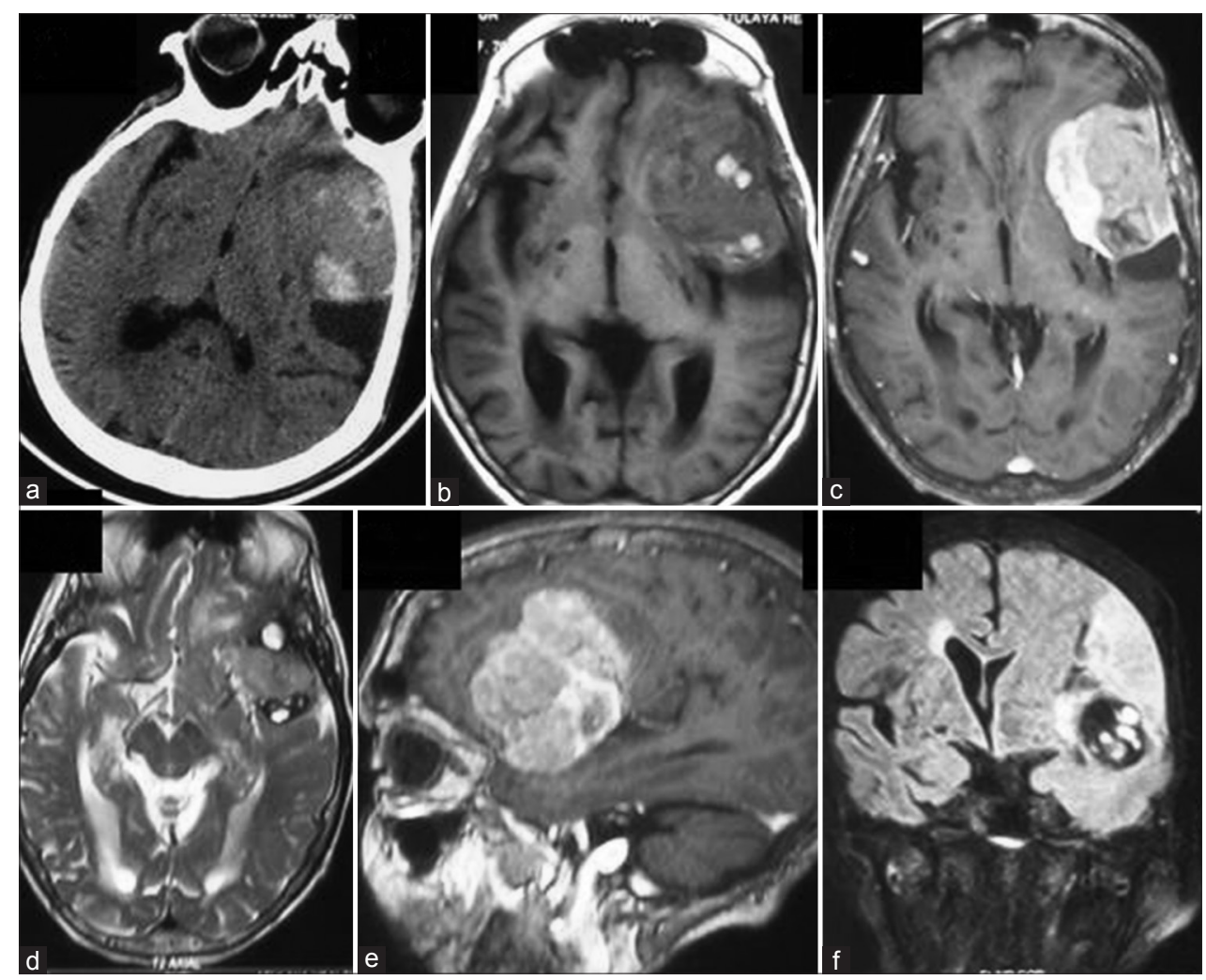

Figure 2: (a) Non contrast computed tomography head demonstrating a largely isointense space-occupying lesion in left fronto-temporal region with areas of hyperintensity. (b-e) Magnetic resonance imaging showing a large lesion in left fronto-temporal area. The lesion was hypodense on T1-weighted imaging with hyperdense areas. On T2-weighted image the lesion was hyperdense with hypodense areas in between. (f) Coronal flair image showing variable density tumor with surrounding edema 

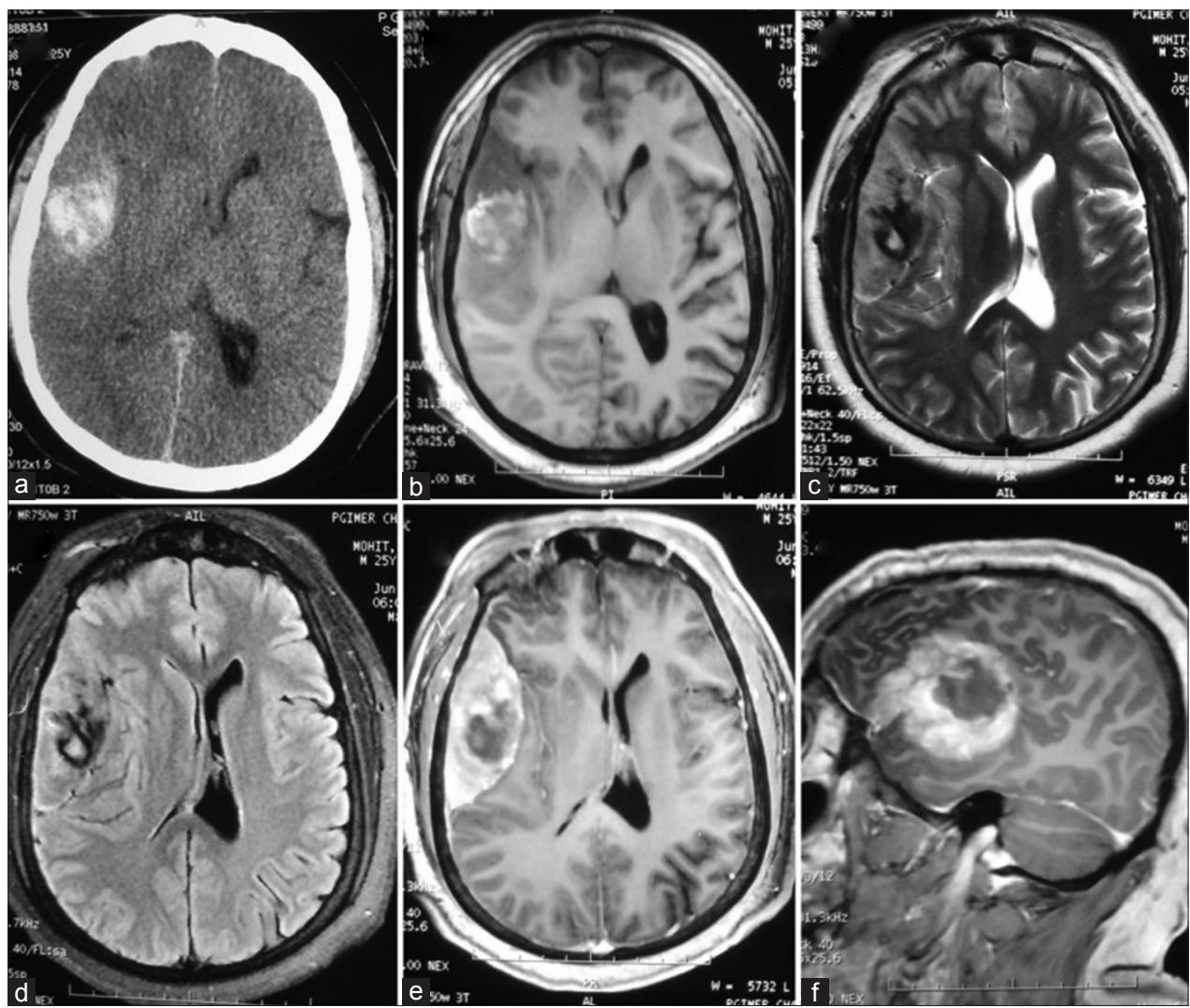

Figure 3: (a) Non contrast computed tomography head showing a hyper intense lesion in right fronto-temporal region with midline shift. (b) Magnetic resonance imaging T1-weighted imaging axial demonstrating an variable intensity extra-axial lesion in right fronto-temporal region. (c and d) T2-weighted image axial variable intensity extra-axial lesion in right fronto-temporal region. (e and f) T1-weighted imaging axial and sagittal: There is intense contrast enhancement with a central area of hypodensity

emergent surgical evacuation of the lesion. ITH may present like a stroke/cerebrovascular accident. Usually, the first radiological investigation in an unconscious patient is NCCT. However, in these cases, NCCT alone can be misleading.

Case 1 was misinterpreted as deep grey matter bleed and did not qualify for operative intervention at first instance. ${ }^{[1]}$ MRI lead us to correct diagnosis and a paradigm shift in management from nonoperative to urgent surgical evacuation.

In case 3, the NCCT was suggestive of EDH and patient would have been taken up for surgery, but the absence of trauma (spontaneous EDH are rare) and pointers toward some underlying neurological disease prompted us for MRI.

ITH in meningiomas is a definite entity and has to be considered in differential diagnosis of stroke, especially in the setting of atypical clinical and radiological findings. The atypical clinical findings that need to be borne in mind are the presence of gradually progressive neurological deficits in the recent past (case 1) or undiagnosed seizures (case 2), "hitherto undiagnosed" headaches in history (case 3) and the subset of patients bearing an intracranial meningioma (being managed conservatively).

The role of antiplatelet therapy (case 1 and 2) causing increased chances of bleed in the tumor is still controversial. There are case reports by Miyazawa et al..$^{[3]}$ and Spektor et al. ${ }^{[4]}$ that have found aspirin prophylaxis as one of the contributing factors. However, definitive evidence for this hypothesis is lacking as no cause and effect relationship has been demonstrated.

Patients diagnosed to have meningoma who are being kept on follow-up (without surgery) have a low but definite risk of intratumoral bleed. No time frame has been suggested from initial presentation to bleed.

The role of aspirin in causing ITH is doubtful. However, an unanswered question remains. Are patients diagnosed to have meningioma who are to receive aspirin prophylaxis are at a higher risk of intratumoral bleeds? Should treatment strategy be tailored for these patients? 


\section{Financial support and sponsorship}

Nil.

\section{Conflicts of interest}

There are no conflicts of interest.

Ashish Aggarwal, Kim Vaiphei ${ }^{1}$, Amey R. Savardekar², Pravin Salunke, Suresh N. Mathuriya ${ }^{3}$

Departments of Neurosurgery and ${ }^{1}$ Histopathology, PGIMER, Chandigarh, ${ }^{2}$ Department of Neurosurgery, NIMHANS, Bengaluru, Karnataka, ${ }^{3}$ Department of Neurosurgery, Medipulse Hospital, Jodhpur, Rajasthan, India

Address for correspondence: Dr. Ashish Aggarwal, Department of Neurosurgery, PGIMER, Chandigarh - 160 012, India. E-mail: aaggarwal_7@yahoo.com

\section{References}

1. Mendelow AD, Gregson BA, Rowan EN, Murray GD, Gholkar A, Mitchell PM; STICH II Investigators. Early surgery versus initial conservative treatment in patients with spontaneous supratentorial lobar intracerebral haematomas (STICH II): A randomised trial. Lancet 2013;382:397-408.

2. Bosnjak R, Derham C, Popović M, Ravnik J. Spontaneous intracranial meningioma bleeding: Clinicopathological features and outcome. J Neurosurg 2005;103:473-84.

3. Miyazawa T, Uozumi Y, Toyooka T, Shima K. Hemorrhage from a falx meningioma after internal use of low-dose aspirin. J Stroke Cerebrovasc Dis 2008;17:325-7.

4. Spektor S, Ashkenazi E, Israel Z. Intracranial haemorrhage from a meningioma in a patient receiving aspirin prophylaxis: A case report. Acta Neurochir (Wien) 1995;134:51-3.

This is an open access article distributed under the terms of the Creative Commons Attribution-NonCommercial-ShareAlike 3.0 License, which allows others to remix, tweak, and build upon the work non-commercially, as long as the author is credited and the new creations are licensed under the identical terms.

\begin{tabular}{|l|l|}
\hline \multicolumn{2}{|c|}{ Access this article online } \\
\hline Quick Response Code: & Website: \\
\hline & www.ruralneuropractice.com \\
\cline { 2 - 3 } & \\
\hline & DOI: \\
\hline
\end{tabular}

How to cite this article: Aggarwal A, Vaiphei K, Savardekar AR, Salunke P, Mathuriya SN. Stroke in three patients due to spontaneous intra tumoral hemorrhage in meningiomas: Lessons learnt. J Neurosci Rural Pract 2016;7:183-6. 